\title{
Case report of primary serous adenocarcinoma of fallopian tube- a diagnostic dilemma
}

\author{
Gayam S. ${ }^{1}$, Babu C. ${ }^{2}$, V.V.S. L. ${ }^{3}$, Maddali S. ${ }^{4}$ \\ ${ }^{1}$ Dr. Susheela Gayam, Department of Obstetrics and Gynaecology, Vijay Marie Hospital, Hyderabad, India, ${ }^{2}$ Dr. Chinna \\ Babu, Dept of Surgical Oncology, Apollo Hospital, Hyderabad, India, ${ }^{3}$ Dr. Lakshmi V.V.S, Department of Obstetrics and \\ Gynaecology, Vijay Marie Hospital, Hyderabad, India, ${ }^{4}$ Dr. Srinivas Maddali, Histopathology \& Cytology Laboratory, \\ Hyderabad, India.
}

Corresponding Author: Dr. Susheela Gayam, Department of Obstetrics and Gynaecology, Vijay Marie Hospital, Hyderabad, India. E-mail: sushgayam@yahoo.com

\begin{abstract}
Historically, primary fallopian tube carcinoma constitutes $0.3 \%$ of all cancers of the female genital tract. But literature suggests that the primary fallopian tube carcinoma may be more common. The true incidence of fallopian tube cancer may be underestimated because of the convention of assigning many of these as ovarian cancer when the site of origin is unclear. The preoperative diagnosis is difficult due to the lack of specific symptoms. Fallopian tube carcinoma clinically behaves like epithelial ovarian cancer and histologically similar to it; thus, the evaluation and treatment are essentially the same. We report here a typical case of primary fallopian tube cancer in a 48 year old female diagnosed as ovarian tumour, preoperatively by radiological imaging but was recognized as fallopian tube carcinoma intraoperatively and was confirmed by histopathology.
\end{abstract}

Keywords: Primary fallopian tube neoplasm, Intra-operative Evaluation, Surgical Procedure, Histopathological Diagnosis, Serous Adenocarcinoma, Staging, Chemotherapy

\section{Introduction}

Primary fallopian tube carcinoma (PFTC) is a very rare gynecologic malignant tumor and accounts for approximately $0.14-1.8 \%$ of female genital malignancies $[1,2]$. The etiology of this tumour is unknown; it is suggested to be associated with chronic tubal inflammation, infertility, tuberculous salpingitis and tubal endometriosis [3].

Similar to ovarian malignancy, a BRCA germ line mutation and TP53 mutation are associated with fallopian tube malignancy $[4,5]$. Many patients, with fallopian tube carcinoma are asymptomatic. The most common clinical features associated with it are abdominal pain, vaginal bleeding, hydrohematorrhea, and palpation of a pelvic mass/an adnexal mass [6]. Due to its rarity, preoperative diagnosis of primary fallopian tube carcinoma is hardly ever made. It is usually misdiagnosed as ovarian carcinoma, tuboovarian abscess or ectopic pregnancy. Sonographic features of the tumor are non-specific and include the presence of a fluid-filled, adnexal structure with a significant solid component, a sausage-shaped mass, a cystic mass with papillary projections within, a cystic mass with cog wheel appearance and an ovoidshaped structure containing an incomplete separation and a highly vascular solid nodule.

More than $80 \%$ of patients have elevated pretreatment serum CA-125 levels, which is useful in follow-up after the definite treatment [3]. The treatment approach is similar to that of ovarian carcinoma, and includes Staging laparotomy, total abdominal hysterectomy and bilateral salpingo-oophorectomy. Surgery is followed by chemotherapy [3].

\section{Case Report}

A 48-year old female, P2L2 who had regular periods, presented with watery vaginal discharge intermittently mixed with blood for 2 months. Past medical or surgical history was nil significant and there was no noteworthy family or personal 


\section{Case Report}

history. General physical and systemic examination was normal. On examination, abdomen was soft and nontender. There was no palpable mass or free fluid noted. Per speculum examination revealed healthy cervix and moist vagina. There was no bleeding through the cervical OS. Bimanual examination revealed normal sized anteverted uterus. A firm and non tender mass of $6 \times 6 \mathrm{~cm}$ was felt separately from the uterus in the left fornix. The other fornices were free and non tender.

Pelvic Sonography showed uterus with normal echotexture measuring $108 \times 63 \times 52 \mathrm{~mm}$, endometrial thickness of $7 \mathrm{~mm}$. Right ovary measured $28 \times 21 \mathrm{~mm}$ and left ovary $28 \times 20 \mathrm{~mm}$ both with normal echo texture. A well-define disoechoec lesion of size $59 \times 54 \times 69 \mathrm{~mm}$ noted in the left adnexal region suggestive of left adnexal complex cyst/endometrioma. Mild free fluid in pelvis was noted. CECT abdomen showed uterus measured $88 \times 52 \times 48 \mathrm{~cm}$; endometrial thickness $7 \mathrm{~mm}$, homogenously enhancing soft tissue mass lesion in the left adnexa with poor delineation of left ovary measures $50 \times 51 \times 55 \mathrm{~mm}(\mathrm{AP} \times \mathrm{TR} \times$ CC) likely suggestive of primary ovarian neoplasm. Noted mild ascites, and few enlarged lymph nodes in the paraaortic and aortocaval region with largest measuring $15 \times 6 \mathrm{~mm}$. Liver was normal in contour and attenuation; no focal or diffuse lesions.

The tumour markers, CA 125 result was 2586U/ml; CA-19-9 and CEA were within normal limits. Routine surgical profile, liver and renal function tests were within normal limits. Chest X-ray and 2DEcho were normal. Pap smear was normal. A diagnostic Curettage was done which showed non secretary endometrium and no atypia. The clinical and radiological findings suggested a left adnexal tumour with grossly elevated CA-125 level.

Staging laparotomy and frozen section were planned in view of a complex ovarian mass. On opening the abdomen, a very obvious solid polypoid growth of $5 \times 5 \times 7 \mathrm{~cm}$ was found arising from the fimbrial end of the left fallopian tube (Figures $1 \& 2$ ).

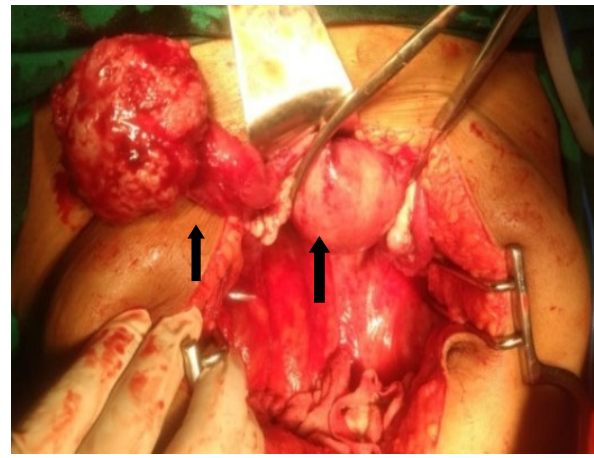

Figure 1: Intraoperative - Solid tumour arising from fimbrial end of the left fallopian tube and normal appearing ovaries (Black Arrows)

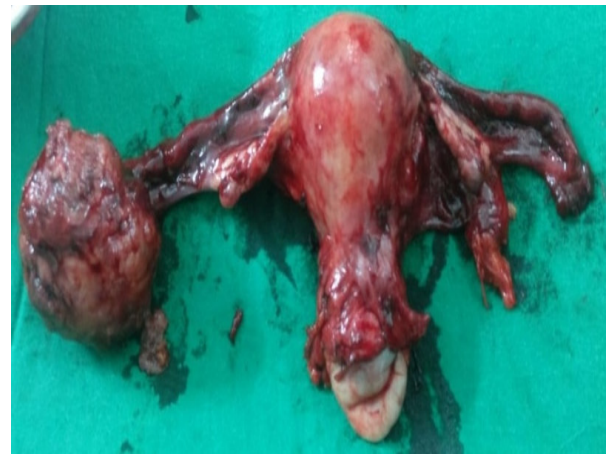

Figure 2: Surgical Specimen of Hysterectomy showing solid growth at fimbrial end of the left fallopian tube and normal Right FT.

About $100 \mathrm{ml}$ of haemorrhagic peritoneal fluid was present in the pelvis and it was aspirated and sent for cytology. The right fallopian tube and both the ovaries appeared normal. Uterovesical fold of peritoneum and peritoneum of pouch of Douglas appeared thickened and congested. Both left and right paracolic gutters, liver surface, the sub-diaphragmatic surfaces, omentum and the rest of viscera appeared normal and no deposits noted. Two paraortic lymph nodes were enlarged to $1.5 \mathrm{x}$ $1 \mathrm{~cm}$ size. The patient underwent staging laparotomy, total abdominal hysterectomy, bilateral salpingo-oophorectomy, total omentectomy and pelvic and paraaotic lymphadenectomy. Anteriorly, uterovesical fold and posteriorly, POD peritonectomy was also done as there were doubtful small deposits. A thorough peritoneal wash was given.

The uterus with adnexae, omentum, the pelvic and paraaortic lymph nodes, and the peritoneal biopsies were sent for histopathological examination. Ascitic fluid cytology was negative for malignant cells. The histpathological examination was indicative of poorly differentiated serous adenocarcinoma of left fallopian tube, solid pattern, (figures $3 \& 4$ ) with tumour present on serosal surface. HPE was suggestive of Histologic grade III and Nuclear grade II.

The uterus, the right fallopian tube and both the ovaries were free of tumour. The endometrium wasnon secretary with no atypia. No lymphovascualr invasion (LVI), or blood vessel invasion (BVI) or tumour on the serosal surface of uterus was detected. Samples from sub diaphragmatic and paracolic sites were negative for malignancy. All the pelvic and paraaortic lymph nodes were also found negative for malignancy. A diagnosis of 'Primary Serous Adenocarcinoma' of the left fallopian tube with HP Grade III, Nuclear grade II and Pathological Stage IC was made. 


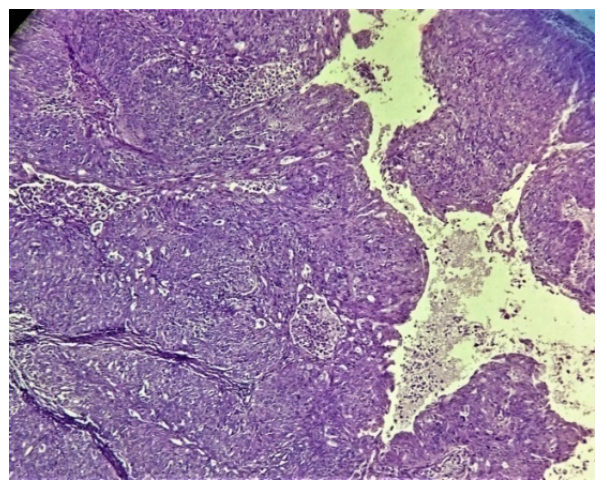

Figure-3: Low power microscopic featuresshowing poorly differentiated serous adenocarcinoma of fallopian tube

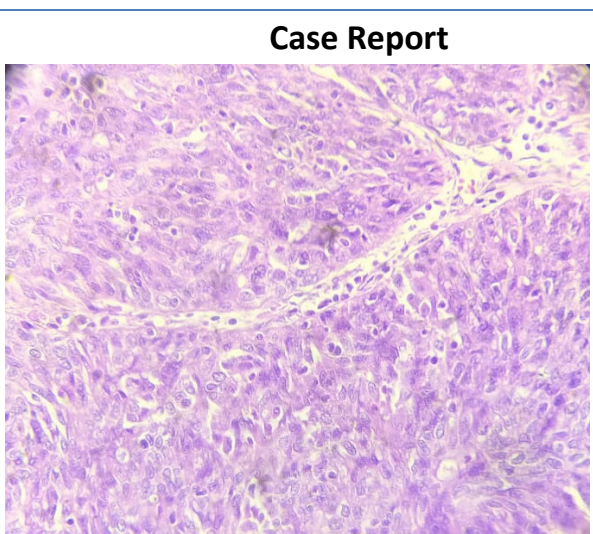

Figure-4: High power microscopic features showing poorly differentiated serous adenocarcinoma of fallopian tube

The patient was discharged seven days after the surgery and was referred for adjuvant chemotherapy. The patient received combination chemotherapy with Paclitaxel and Carboplatin. She tolerated all the cycle. Patent is under regular follow up. In this case, the pre-operative diagnosis made by the clinical and radiological evaluation was incorrect. Fallopian tube carcinomas are difficult to diagnose preoperatively due to infrequency and clinically silent course of this neoplasm.

\section{Discussion}

Primary fallopian tube cancer resembles epithelial ovarian cancers clinically and histologically [7]. Primary fallopian tube cancer is the rarest malignancy of the female genital tract and commonly occurs between fourth and sixth decades of life. It was first described by Renand in 1897 [8]. Rokitansky recorded the first microscopic description in 1861 and Orthman presented a first case report in 1888 [9]. Due to its rarity, preoperative diagnosis of primary fallopian tube carcinoma is seldom made. Vaginal discharge or bleeding is the most common symptom reported by patients with tubal carcinoma and is documented in more than $50 \%$ of patients.

Though this patient presented clinically with watery discharge and a pelvic mass but radiologically it was diagnosed as ovarian tumour. It is also cited in the literature that due to its infrequency, it is typically misdiagnosed as ovarian carcinoma. 3 The preoperative diagnosis of primary fallopian tube cancer is extremely rare [10]. The rate of preoperative diagnosis was in the range of $0 \%-10 \%$, and up to $50 \%$ are missed intraoperatively $[11,12]$. It was noted in this patient a very high levels of CA 125 preoperatively. $80 \%$ of patients with PFTC do have elevated pretreatment serum levels of CA 125 [13]. Surgery is the treatment of choice for PFTC and is similar to that for ovarian carcinomacytoreductive surgery with the removal of the tumour as much as possible.

The procedure of choice is total abdominal hysterectomy, bilateral salpingo-oophorectomy, omentectomy, selective pelvic and para-aortic lymphadenectomy for any stage of fallopian tube carcinoma $[14,15]$. This patient also underwent staging laparotomy, total abdominal hysterectomy and total omentectomy and pelvic and paraaortic lymphadenectomy and the diagnosis of PFTC was confirmed by histopathology and Stage IC wasmade. Postoperative adjuvant chemotherapy with platinum based combination is the most commonly used therapy for these patients, similar to epithelial ovarian cancer patients [16]. The reported 5-year survival rate for patients with stage I disease is about $65 \%$.

\section{Conclusion}

Primary fallopian tube cancer is a rare tumour which accounts for $<1 \%$ of all the female genital tract cancers. Primary fallopian Tubal cancers are seen normally in postmenopausal women, in the fifth and sixth decades. Latzko' classic triad of symptoms and signs associated with fallopian tube cancer is a prominent watery vaginal discharge (ie hydrops tubaeprofluens), Pelvic pain, and a pelvic mass. This triad is noted in fewer than $15 \%$ of patients. The symptoms may be rather vague and non specific. Most commonly involved site is the ampullary part of the fallopian tube. The most common Histologic variant is papillary serous carcinoma. Because the lumen and fimbria of the fallopian tube have access to the peritoneal cavity, fallopian tube carcinomas frequently involve the omentum and peritoneal cavity at the time of presentation. In histologic features and behavior, fallopian tube carcinoma is similar to ovarian cancer; thus, the evaluation and treatment are essentially the same. Surgery is the treatment of choice. Exploratory laparotomy is necessary to remove the primary tumor, to stage the disease, and to resect metastases. In view of the early lymphatic spread, the role of the routine lymph node removal is mandatory in primary fallopian tube cancer. Cytoreductive surgery followed by adequate cycles of 
platinum based combination chemotherapy is an important strategy to improve patients' prognosis.

Competing Interests: The authors have declared that no competing interests exist.

Consent of Patient: Written informed consent was obtained from the patient for publication of this case report and the accompanying images. A copy of the written consent is available for review by the Editor-inChief of this journal.

Funding: Nil, Conflict of interest: Nil Permission from IRB: Yes

\section{References}

1. Kalampokas E, Kalampokas T, Tourountous I. Primary fallopian tube carcinoma. Eur J Obstet Gynecol Reprod Biol. 2013 Jul;169(2):155-61. doi: 10.1016/j.ejogrb.2013. 03.023. Epub 2013 Apr 23.

2. Lau HY, Chen YJ, Yen MS, et al. Primary fallopian tube carcinoma: a clinicopathologic analysis and literature review. J Chin Med Assoc. 2013; 76 (10):583-7. https://doi. org/10. 1016/j.jcma.2013.06.010 .

3. Mladenovic-Segedi L. Primary fallopian tube carcinoma. Med Pregled. 2009;62(1-2):31-6. https://doi. org/10. 2298/MPNS0902031M . PMid: 19514598.

4. Howitt BE, Hanamornroongruang S, Lin DI, et al. Evidence for a dualistic model of high-grade serous carcinoma: BRCA mutation status, histology, and tubal intraepithelial carcinoma. Am J Surg Pathol. 2015 Mar;39 (3): 287-93. doi: 10.1097/PAS.0000000000000369.

5. Quartuccio SM, Karthikeyan S, Eddie SL, et al. Mutant p53 expression in fallopian tube epithelium drives cell migration. Int J Cancer. 2015 Oct 1;137(7):1528-38. doi: 10.1002/ijc.29528. Epub 2015 Apr 11.

6. Wang PH, Yuan CC, Chao HT, et al. Prognosis of primary fallopian tube adenocarcinoma: report of 25 patients. Eur J Gynaecol Oncol. 1998;19(6):571-4.

7. Rexhepi M, Trajkovska E, Ismaili H, Primary Fallopian Tube Carcinoma: A Case Report and Literature Review.

\section{Case Report}

Open Access Maced J Med Sci. 2017 May 20;5(3):344348. doi: 10.3889/oamjms.2017.044. eCollection 2017 Jun 15.

8. Nordin AJ. Primary carcinoma of the fallopian tube: a 20-year literature review. Obstet Gynecol Surv. 1994 May;49(5):349-61.

9. Kalyani R, Kumar ML, Srikantia SH. et al. Primary adenocarcinoma of fallopian tube--a case report. Indian $\mathrm{J}$ Pathol Microbiol. 2005 Apr;48(2):219-21.

10. Koo Yu-Jin K, Kyoung-Shil I, Yong-Soon K. Primary fallopian tube carcinoma: a clinicopathological analysis of a rare entity. International Journal of Clinical Oncology. 2011;16(1):45-49. https://doi.org/10.1007/s10147-0100128-8 .

11. Huber-Buchholz MM, Buchholz NP, Staehelin J. Analysis of 23 cases of primary carcinoma of the fallopian tube over 50 years. J Obstet Gynaecol Res. 1996 Jun; 22(3):193-9.

12. Horng HC, Teng SW, Huang BS, et al. Primary fallopian tube cancer: domestic data and up-to-date review. Taiwan J Obstet Gynecol. 2014 Sep;53(3):287-92. doi: 10.1016/j.tjog.2014.07.003.

13. Chaudhry S, Hussain R, Zuberi MM, et al. Rare primary fallopian tube carcinoma; a gynaecologist's dilemma. J Pak Med Assoc. 2016 Jan;66(1):107-10.

14. Berek JS, Crum C, Friedlander M. Cancer of the ovary, fallopian tube, and peritoneum. Int J Gynaecol Obstet. 2015 Oct;131 Suppl 2:S111-22. doi: 10.1016/j. ijgo.2015.06.007.

15. Gomes FV, Dias JL, Lucas R, Cunha TM. Primary fallopian tube carcinoma: review of MR imaging findings. Insights Imaging. 2015;6:431-439. https://doi.org/10. 1007/s13244-015-0416-y.

16. Hariprasad P, S H, Srinivas T, et al. Primary bilateral fallopian tube carcinoma the report of a single case with review of the literature. J Clin Diagn Res. 2013 May;7 (5):930-2. doi: 10.7860/JCDR/2013/5483.2980. Epub 2013 Mar 7.

\section{How to cite this article?}

Gayam S, Babu C, V.V.S. L, Maddali S. Case report of primary serous adenocarcinoma of fallopian tube- a diagnostic dilemma.Obs Rev:J obstet Gynecol 2018;4(4):73-76.doi:10.17511/joog.2018.i04.01. 